\title{
ON OSCILLATION OF A FOOD-LIMITED POPULATION MODEL WITH TIME DELAY
}

\author{
LEONID BEREZANSKY AND ELENA BRAVERMAN
}

Received 1 October 2001

For a scalar nonlinear delay differential equation $\dot{N}(t)=r(t) N(t)(K-N(h(t)))$ / $(K+s(t) N(g(t))), r(t) \geq 0, K>0, h(t) \leq t, g(t) \leq t$ and some generalizations of this equation, we establish explicit oscillation and nonoscillation conditions. Coefficient $r(t)$ and delays are not assumed to be continuous.

\section{Introduction}

The delay logistic equation

$$
\dot{N}(t)=r(t) N(t)\left(1-\frac{N(h(t))}{K}\right), \quad h(t) \leq t,
$$

is known as Hutchinson's equation, if $r$ and $K$ are positive constants and $h(t)=$ $t-\tau$ for a positive constant $\tau$. Hutchinson's equation has been investigated by several authors (see, e.g., $[13,14,18,23]$ ). Delay logistic equation (1.1) was studied by Gopalsamy and Zhang $[7,25]$ who gave sufficient conditions for the oscillation and the nonoscillation of (1.1). Publications $[1,2,3,4,5,6,10,12,15,16$, $17,19,22,24]$ are devoted to various generalizations of logistic equation (1.1).

In 1963, Smith [20] proposed an alternative to the logistic equation for a food-limited population

$$
\dot{N}(t)=r N(t) \frac{K-N(t)}{K+c r N(t)}, \quad t \geq 0
$$

Here $N, r$, and $K$ are the mass of the population, the rate of increase with unlimited food, and value of $N$ at saturation, respectively. The constant $1 / c$ is the rate of replacement of mass in the population at saturation (this includes both the replacement of metabolic loss and of dead organisms). 
In $[8,9,11]$, Gopalsamy, Kulenovic, Ladas, Grove, and Qian considered the autonomous delay food-limited equation

$$
\dot{N}(t)=r N(t) \frac{K-N(t-\tau)}{K+c r N(t-\tau)}, \quad t \geq 0 .
$$

So and Yu [21] investigated stability properties of the following nonlinear differential equation with a constant delay:

$$
\dot{N}(t)=r(t) N(t) \frac{K-N^{l}(t-\tau)}{K+s(t) N^{l}(t-\tau)}, \quad t \geq 0,
$$

which is a generalization of food-limited equations (1.2) and (1.3).

In this paper, we consider oscillation properties of a nonautonomous foodlimited equation with a nonconstant delay

$$
\dot{N}(t)=r(t) N(t) \frac{K-N(h(t))}{K+s(t) N(g(t))}, \quad t \geq 0, h(t) \leq t, g(t) \leq t,
$$

which also generalizes (1.3). We compare oscillation properties of (1.5) and some linear delay differential equations. As a corollary, we obtain explicit oscillation and nonoscillation conditions for (1.5). For the autonomous equation (1.3), our conditions and the known ones in [8] coincide.

We also consider two generalizations of (1.5), the first one is (1.4) with a nonconstant delay and the second one is (1.5) with several delays.

Our proof of the main result is based on some application of Schauder's fixedpoint theorem which was employed for a generalized logistic equation in [4]. According to this method, the differential equation is transformed into an operator equation

$$
u=A u B u
$$

where operator $A$ is a monotone increasing operator and $B$ is a monotone decreasing one. We prove that there exist two functions $v, w, 0 \leq v(t) \leq w(t)$, such that $v(t) \leq(A v)(t)(B w)(t), w(t) \geq(A w)(t)(B v)(t)$. Then operator $T u=A u B u$ acts in the interval $v(t) \leq u(t) \leq w(t)$ and therefore, we can use Schauder's fixedpoint theorem. Functions $w$ and $v$ are the limits of two sequences $\left\{w_{n}\right\}$ and $\left\{v_{n}\right\}$, respectively, and for the construction of the first approximation $w_{1}$, we apply a positive solution of some linear delay differential equation.

The paper is organized as follows. In Sections 2 and 3, we consider an equation which is obtained from (1.5) by the following substitution:

$$
x(t)=\frac{N(t)}{K}-1 .
$$

On the base of these results, in Section 4, we investigate generalized delay logistic equation (1.5). 


\section{Preliminaries}

Consider a scalar delay differential equation

$$
\dot{x}(t)=-r(t) x(h(t)) \frac{1+x(t)}{1+s(t)[1+x(g(t))]}, \quad t \geq 0,
$$

under the following assumptions:

(A1) $r(t)$ and $s(t)$ are Lebesgue measurable locally essentially bounded functions, $r(t) \geq 0$ and $s(t) \geq 0$,

(A2) $h, g:[0, \infty) \rightarrow \mathbb{R}$ are Lebesgue measurable functions, $h(t) \leq t, g(t) \leq t$, $\lim _{t \rightarrow \infty} h(t)=\infty$, and $\lim _{t \rightarrow \infty} g(t)=\infty$.

Together with (2.1), we consider for each $t_{0} \geq 0$ an initial value problem

$$
\begin{gathered}
\dot{x}(t)=-r(t) x(h(t)) \frac{1+x(t)}{1+s(t)[1+x(g(t))]}, \quad t \geq t_{0}, \\
x(t)=\varphi(t), \quad t<t_{0}, x\left(t_{0}\right)=x_{0} .
\end{gathered}
$$

We also assume that the following hypothesis holds:

(A3) $\varphi:\left(-\infty, t_{0}\right) \rightarrow \mathbb{R}$ is a Borel measurable bounded function.

Definition 2.1. An absolutely continuous, in each interval $\left[t_{0}, b\right]$, function $x$ : $\mathbb{R} \rightarrow \mathbb{R}$ is called a solution of problem (2.2) and (2.3), if it satisfies (2.2) for almost all $t \in\left[t_{0}, \infty\right)$ and equalities (2.3) for $t \leq t_{0}$.

Equation (2.1) has a nonoscillatory solution if it has an eventually positive or an eventually negative solution. Otherwise, all solutions of (2.1) are oscillatory.

We present here Lemma 2.2 which will be used in the proof of the main results.

Consider the linear delay differential equation

$$
\dot{x}(t)+r(t) x(h(t))=0, \quad t \geq 0 .
$$

Lemma 2.2 (see [12]). Let (A1) and (A2) hold for (2.4). Then the following hypotheses are equivalent:

(1) the differential inequality

$$
\dot{x}(t)+r(t) x(h(t)) \leq 0, \quad t \geq 0,
$$

has an eventually positive solution; 
58 Oscillation of a food-limited model

(2) there exists $t_{0} \geq 0$ such that the inequality

$$
u(t) \geq r(t) \exp \left\{\int_{h(t)}^{t} u(s) d s\right\}, \quad t \geq t_{0} ; \quad u(t)=0, \quad t<t_{0},
$$

has a nonnegative locally integrable solution;

(3) equation (2.4) has a nonoscillatory solution.

If

$$
\lim _{t \rightarrow \infty} \sup \int_{h(t)}^{t} r(s) d s<\frac{1}{e}
$$

then (2.4) has a nonoscillatory solution. If

$$
\liminf _{t \rightarrow \infty} \int_{h(t)}^{t} r(s) d s>\frac{1}{e},
$$

then all the solutions of (2.4) are oscillatory.

\section{Oscillation conditions}

In this section and Section 4, we assume that (A1), (A2), and (A3) hold and consider only such solutions of (2.1) for which the following condition holds:

$$
1+x(t)>0
$$

We begin with the following lemma.

Lemma 3.1. Suppose

$$
\int_{0}^{\infty} \frac{r(t)}{1+s(t)} d t=\infty
$$

and $x(t)$ is a nonoscillatory solution of (2.1). Then $\lim _{t \rightarrow \infty} x(t)=0$.

Proof. Suppose first $x(t)>0, t \geq t_{1}$. Then there exists $t_{2} \geq t_{1}$ such that

$$
h(t) \geq t_{1}, \quad g(t) \geq t_{1},
$$

for $t \geq t_{2}$. Denote

$$
u(t)=-\frac{\dot{x}(t)}{x(t)}, \quad t \geq t_{2}
$$

Then $u(t) \geq 0, t \geq t_{2}$. Substitute

$$
x(t)=x\left(t_{2}\right) \exp \left\{-\int_{t_{2}}^{t} u(s) d s\right\}, \quad t \geq t_{2}
$$


in (2.1). After some transformations, we have the following equation:

$$
u(t)=\frac{r(t)}{1+s(t)} \exp \left\{\int_{h(t)}^{t} u(s) d s\right\} \frac{[1+s(t)]\left[1+c \exp \left\{-\int_{t_{2}}^{t} u(s) d s\right\}\right]}{1+s(t)\left[1+c \exp \left\{-\int_{t_{2}}^{g(t)} u(s) d s\right\}\right]}
$$

where $h(t) \leq t, g(t) \leq t, t \geq t_{2}$, and $c=x\left(t_{2}\right)>0$.

Hence,

$$
u(t) \geq \frac{r(t)}{1+s(t)} \frac{1+s(t)}{(1+c)(1+s(t))}=\frac{r(t)}{(1+c)(1+s(t))}
$$

Then, by (3.2), $\int_{t_{2}}^{\infty} u(t) d t=\infty$.

Now suppose $-1<x(t)<0, t \geq t_{1}$. Then there exists $t_{2} \geq t_{1}$ such that (3.3) holds for $t \geq t_{2}$. Suppose $u(t)$ is denoted by (3.4) and $c=x\left(t_{2}\right)$. Then $u(t) \geq 0$, $-1<c<0$. Substitute (3.5) into (2.1). Thus (3.6) yields

$$
u(t) \geq \frac{r(t)}{1+s(t)} \frac{(1+c)(1+s(t))}{1+s(t)}=\frac{r(t)(1+c)}{1+s(t)} .
$$

Then again $\int_{t_{2}}^{\infty} u(t) d t=\infty$.

Equation (3.5) implies $\lim _{t \rightarrow \infty} x(t)=0$.

Theorem 3.2. Suppose (3.2) holds and for some $\epsilon>0$, all solutions of the linear equation

$$
\dot{x}(t)+(1-\epsilon) \frac{r(t)}{1+s(t)} x(h(t))=0
$$

are oscillatory. Then all solutions of (2.1) are oscillatory.

Proof. First suppose $x(t)$ is an eventually positive solution of (2.1). Lemma 3.1 implies that there exists $t_{1} \geq 0$ such that $0<x(t)<\epsilon$ for $t \geq t_{1}$. We suppose (3.3) holds for $t \geq t_{2} \geq t_{1}$. For $t \geq t_{2}$, we have

$$
\frac{[1+s(t)](1+x(t))}{1+s(t)[1+x(g(t))]} \geq \frac{1+s(t)}{1+s(t)(1+\epsilon)} \geq \frac{1+s(t)}{(1+\epsilon)(1+s(t))}=\frac{1}{1+\epsilon} \geq 1-\epsilon
$$

Equation (2.1) implies

$$
\dot{x}(t)+(1-\epsilon) \frac{r(t)}{1+s(t)} x(h(t)) \leq 0, \quad t \geq t_{2} .
$$

Lemma 2.2 yields that (3.9) has a nonoscillatory solution. We have a contradiction. 
Now suppose $-\epsilon<x(t)<0$ for $t \geq t_{1}$ and (3.3) holds for $t \geq t_{2} \geq t_{1}$. Then for $t \geq t_{2}$

$$
\frac{[1+s(t)](1+x(t))}{1+s(t)[1+x(g(t))]} \geq \frac{(1+s(t))(1-\epsilon)}{1+s(t)}=1-\epsilon .
$$

Hence, (3.9) has a nonoscillatory solution and we again obtain a contradiction which completes the proof.

Corollary 3.3. If

$$
\liminf _{t \rightarrow \infty} \int_{h(t)}^{t} \frac{r(\tau)}{1+s(\tau)} d \tau>\frac{1}{e},
$$

then all solutions of (2.1) are oscillatory.

TheOREM 3.4. Suppose for some $\epsilon>0$ there exists a nonoscillatory solution of the linear delay differential equation

$$
\dot{x}(t)+(1+\epsilon) \frac{r(t)}{1+s(t)} x(h(t))=0 .
$$

Then there exists a nonoscillatory solution of (2.1).

Proof. Lemma 2.2 implies that there exist $t_{0} \geq 0$ and $w_{0}(t) \geq 0, t \geq t_{0} ; w_{0}(t)=0$, $t \leq t_{0}$ such that

$$
w_{0}(t) \geq(1+\epsilon) \frac{r(t)}{1+s(t)} \exp \left\{\int_{h(t)}^{t} w_{0}(s) d s\right\} .
$$

Suppose $0<c<\epsilon$ and consider two sequences:

$$
\begin{aligned}
& w_{n}(t)=r(t) \exp \left\{\int_{h(t)}^{t} w_{n-1}(s) d s\right\} \frac{1+c \exp \left\{-\int_{t_{0}}^{t} v_{n-1}(s) d s\right\}}{1+s(t)\left(1+c \exp \left\{-\int_{t_{0}}^{g(t)} w_{n-1}(s) d s\right\}\right)}, \\
& v_{n}(t)=r(t) \exp \left\{\int_{h(t)}^{t} v_{n-1}(s) d s\right\} \frac{1+c \exp \left\{-\int_{t_{0}}^{t} w_{n-1}(s) d s\right\}}{1+s(t)\left(1+c \exp \left\{-\int_{t_{0}}^{g(t)} v_{n-1}(s) d s\right\}\right)},
\end{aligned}
$$

where $w_{0}$ was defined above and $v_{0} \equiv 0$. We have

$$
\begin{aligned}
w_{1}(t) & =\frac{r(t)}{1+s(t)} \exp \left\{\int_{h(t)}^{t} w_{0}(s) d s\right\} \frac{(1+s(t))(1+c)}{1+s(t)\left(1+c \exp \left\{-\int_{t_{0}}^{g(t)} w_{0}(s) d s\right\}\right)} \\
& \leq \frac{r(t)}{1+s(t)} \exp \left\{\int_{h(t)}^{t} w_{0}(s) d s\right\} \frac{(1+s(t))(1+\epsilon)}{1+s(t)} \leq w_{0}(t) .
\end{aligned}
$$

It is evident that $v_{1}(t) \geq v_{0}(t), w_{0}(t) \geq v_{0}(t)$. 
Hence by induction,

$$
0 \leq w_{n}(t) \leq w_{n-1}(t) \leq \cdots \leq w_{0}(t), \quad v_{n}(t) \geq v_{n-1}(t) \geq \cdots \geq v_{0}(t)=0,
$$

and $w_{n}(t) \geq v_{n}(t)$.

There exist pointwise limits of nonincreasing nonnegative sequence $w_{n}(t)$ and of nondecreasing sequence $v_{n}(t)$. If we denote $w(t)=\lim _{n \rightarrow \infty} w_{n}(t)$ and $v(t)=\lim _{n \rightarrow \infty} v_{n}(t)$, then by the Lebesgue Convergence theorem, we conclude that

$$
\begin{aligned}
& w(t)=r(t) \exp \left\{\int_{h(t)}^{t} w(s) d s\right\} \frac{1+c \exp \left\{-\int_{t_{0}}^{t} v(s) d s\right\}}{1+s(t)\left(1+c \exp \left\{-\int_{t_{0}}^{g(t)} w(s) d s\right\}\right)}, \\
& v(t)=r(t) \exp \left\{\int_{h(t)}^{t} v(s) d s\right\} \frac{1+c \exp \left\{-\int_{t_{0}}^{t} w(s) d s\right\}}{1+s(t)\left(1+c \exp \left\{-\int_{t_{0}}^{g(t)} v(s) d s\right\}\right)} .
\end{aligned}
$$

We fix $b \geq t_{0}$ and define operator $T: L_{\infty}\left[t_{0}, b\right] \rightarrow: L_{\infty}\left[t_{0}, b\right]$ by the following equality:

$$
(T u)(t)=r(t) \exp \left\{\int_{h(t)}^{t} u(s) d s\right\} \frac{1+c \exp \left\{-\int_{t_{0}}^{t} u(s) d s\right\}}{1+s(t)\left(1+c \exp \left\{-\int_{t_{0}}^{g(t)} u(s) d s\right\}\right)}
$$

where $L_{\infty}\left[t_{0}, b\right]$ is the space of all essentially bonded on $\left[t_{0}, b\right]$ functions with the usual norm.

For every function $u$ from the interval $v \leq u \leq w$, we have $v \leq T u \leq w$. The result of [4, Lemma 3] implies that operator $T$ is a compact operator on the space $L_{\infty}\left[t_{0}, b\right]$. Then by Schauder's fixed-point theorem there exists a nonnegative solution of equation $u=T u$.

Denote

$$
x(t)= \begin{cases}c \exp \left\{-\int_{t_{0}}^{t} u(s) d s\right\}, & t \geq t_{0} \\ 0, & t<t_{0}\end{cases}
$$

Then $x(t)$ is a nonoscillatory solution of (2.1) which completes the proof.

Corollary 3.5. If

$$
\lim _{t \rightarrow \infty} \sup _{h_{(t)}}^{t} \frac{r(\tau)}{1+s(\tau)} d \tau<\frac{1}{e}
$$

then (2.1) has a nonoscillatory solution. 


\section{Main results}

Now consider the delay logistic equation (1.5) where the parameters of this equation satisfy conditions (A1) and (A2), $K>0$, and the initial function $\psi$ satisfies (A3). There exists a unique solution of (1.5) with the initial condition

$$
N(t)=\psi(t), \quad t<t_{0}, \quad N\left(t_{0}\right)=y_{0} .
$$

In this section, we assume that the following additional condition holds:

(A4) $y_{0}>0, \psi(t) \geq 0, t<t_{0}$.

Then as in the autonomous case $[8,12]$ the solution of $(1.5)$ and (4.1) is positive.

A positive solution $N$ of (1.5) is said to be oscillatory about $K$ if there exists a sequence $t_{n}, t_{n} \rightarrow \infty$, such that $N\left(t_{n}\right)-K=0, n=1,2, \ldots ; N$ is said to be nonoscillatory about $K$ if there exists $t_{0} \geq 0$ such that $|N(t)-K|>0$ for $t \geq t_{0}$. A solution $N$ is said to be eventually positive (eventually negative) about $K$ if $N-K$ is eventually positive (eventually negative).

Suppose $N$ is a positive solution of (1.5) and define $x$ as $x=N / K-1$. Then $x$ is a solution of (2.1) such that $1+x>0$.

Hence, oscillation (or nonoscillation) of $N$ about $K$ is equivalent to oscillation (nonoscillation) of $x$.

By applying Theorems 3.2 and 3.4, we obtain the following results for (1.5).

Theorem 4.1. Suppose (3.2) holds and for some $\epsilon>0$, all solutions of the linear equation

$$
\dot{x}(t)+(1-\epsilon) \frac{r(t)}{1+s(t)} x(h(t))=0
$$

are oscillatory. Then all solutions of (1.5) are oscillatory about $\mathrm{K}$.

Theorem 4.2. Suppose for some $\epsilon>0$ there exists a nonoscillatory solution of the linear delay differential equation

$$
\dot{x}(t)+(1+\epsilon) \frac{r(t)}{1+s(t)} x(h(t))=0 .
$$

Then there exists a nonoscillatory about $K$ solution of (1.5).

Corollary 4.3. If

$$
\liminf _{t \rightarrow \infty} \int_{h(t)}^{t} \frac{r(\tau)}{1+s(\tau)} d \tau>\frac{1}{e}
$$


then all solutions of (1.5) are oscillatory about $K$. If

$$
\lim _{t \rightarrow \infty} \sup \int_{h(t)}^{t} \frac{r(\tau)}{1+s(\tau)} d \tau<\frac{1}{e}
$$

then (1.5) has a nonoscillatory about $K$ solution.

Now consider a generalized delay food-limited equation

$$
\dot{N}(t)=r(t) N(t) \frac{K-N(h(t))|N(h(t))|^{l-1}}{K+s(t) N(g(t))|N(g(t))|^{l-1}},
$$

where $l>0$ and for the other parameters conditions (A1) and (A2) hold.

After the substitution $y(t)=N(t)|N(t)|^{l-1}$, (4.6) turns into the following one:

$$
\dot{y}(t)=\operatorname{lr}(t) y(t) \frac{K-y(h(t))}{K+s(t) y(g(t))} .
$$

It is easy to see that (4.6) has a nonoscillatory about $K^{1 / l}$ solution if and only if (4.7) has a nonoscillatory about $K$ solution.

For (4.7), Theorems 4.1, 4.2 and their corollary can be applied. Hence, we have the following results.

Theorem 4.4. Suppose (3.2) holds and for some $\epsilon>0$, all solutions of the linear equation

$$
\dot{x}(t)+(1-\epsilon) \frac{\operatorname{lr}(t)}{1+s(t)} x(h(t))=0
$$

are oscillatory. Then all solutions of (4.6) are oscillatory about $K^{1 / l}$.

Theorem 4.5. Suppose for some $\epsilon>0$ there exists a nonoscillatory solution of linear delay differential equation

$$
\dot{x}(t)+(1+\epsilon) \frac{\operatorname{lr}(t)}{1+s(t)} x(h(t))=0 .
$$

Then there exists a nonoscillatory about $K^{1 / l}$ solution of (4.6).

Corollary 4.6. If

$$
\liminf _{t \rightarrow \infty}^{t} \frac{\operatorname{lr}(\tau)}{h(t)} d \tau>\frac{1}{e}
$$


64 Oscillation of a food-limited model

then all solutions of (4.6) are oscillatory about $K^{1 / l}$. If

$$
\limsup _{t \rightarrow \infty} \int_{h(t)}^{t} \frac{\operatorname{lr}(\tau)}{1+s(\tau)} d \tau<\frac{1}{e}
$$

then there exists a nonoscillatory about $K^{1 / l}$ solution of (4.6).

Now consider a food-limited equation with several delays

$$
\dot{N}(t)=\sum_{k=1}^{m} r_{k}(t) N(t) \frac{K-N\left(h_{k}(t)\right)}{K+s_{k}(t) N\left(g_{k}(t)\right)},
$$

where the parameters of this equation satisfy conditions (A1) and (A2), $K>0$, and the initial function $\psi$ satisfies (A3).

Similar to the case $m=1$, the following generalizations of Theorems 4.1 and 4.2 can be obtained.

Theorem 4.7. Suppose (3.2) holds and for some $\epsilon>0$ all solutions of the linear equation

$$
\dot{x}(t)+(1-\epsilon) \sum_{k=1}^{m} \frac{r_{k}(t)}{1+s_{k}(t)} x\left(h_{k}(t)\right)=0
$$

are oscillatory. Then all solutions of (4.12) are oscillatory about $K$.

TheOrem 4.8. Suppose for some $\epsilon>0$ there exists a nonoscillatory solution of the linear delay differential equation

$$
\dot{x}(t)+(1+\epsilon) \sum_{k=1}^{m} \frac{r_{k}(t)}{1+s_{k}(t)} x(h(t))=0 .
$$

Then there exists a nonoscillatory about $K$ solution of (4.12).

Corollary 4.9. If

$$
\liminf _{t \rightarrow \infty} \sum_{k=1}^{m} \int_{\max h_{k}(t)}^{t} \frac{r_{k}(\tau)}{1+s_{k}(\tau)} d \tau>\frac{1}{e},
$$

then all solutions of (4.12) are oscillatory about K. If

$$
\limsup _{t \rightarrow \infty} \sum_{k=1}^{m} \int_{\min h_{k}(t)}^{t} \frac{r_{k}(\tau)}{1+s_{k}(\tau)} d \tau<\frac{1}{e}
$$

then (4.12) has a nonoscillatory about K solution. 


\section{References}

[1] W. G. Aiello, The existence of nonoscillatory solutions to a generalized, nonautonomous, delay logistic equation, J. Math. Anal. Appl. 149 (1990), no. 1, 114-123.

[2] L. Berezansky and E. Braverman, On oscillation of a logistic equation with several delays, J. Comput. Appl. Math. 113 (2000), no. 1-2, 255-265.

[3] Oscillation properties of a logistic equation with several delays, J. Math. Anal. Appl. 247 (2000), no. 1, 110-125.

[4] On oscillation of a generalized logistic equation with several delays, J. Math. Anal. Appl. 253 (2001), no. 2, 389-405.

[5] M.-P. Chen, B. S. Lalli, and J. S. Yu, Oscillation and global attractivity in a multiplicative delay logistic equation, Differential Equations Dynam. Systems 5 (1997), no. 1, 75-83.

[6] L. H. Erbe, Q. Kong, and B. G. Zhang, Oscillation Theory for Functional-Differential Equations, Monographs and Textbooks in Pure and Applied Mathematics, vol. 190, Marcel Dekker, New York, 1995.

[7] K. Gopalsamy, Stability and Oscillations in Delay Differential Equations of Population Dynamics, Mathematics and its Applications, vol. 74, Kluwer Academic Publishers Group, Dordrecht, 1992.

[8] K. Gopalsamy, M. R. S. Kulenović, and G. Ladas, Time lags in a "food-limited" population model, Appl. Anal. 31 (1988), no. 3, 225-237.

[9] Environmental periodicity and time delays in a "food-limited" population model, J. Math. Anal. Appl. 147 (1990), no. 2, 545-555.

[10] S. R. Grace, I. Györi, and B. S. Lalli, Necessary and sufficient conditions for the oscillations of a multiplicative delay logistic equation, Quart. Appl. Math. 53 (1995), no. 1, 69-79.

[11] E. A. Grove, G. Ladas, and C. Qian, Global attractivity in a "food-limited" population model, Dynam. Systems Appl. 2 (1993), no. 2, 243-249.

[12] I. Győri and G. Ladas, Oscillation Theory of Delay Differential Equations, The Clarendon Press Oxford University Press, New York, 1991.

[13] G. S. Jones, On the nonlinear differential-difference equation $f^{\prime}(x)=-\alpha f(x-1)\{1+$ $f(x)\}$, J. Math. Anal. Appl. 4 (1962), 440-469.

[14] S. Kakutani and L. Markus, On the non-linear difference-differential equation $y^{\prime}(t)=$ $[A-B y(t-\tau)] y(t)$, Contributions to the Theory of Nonlinear Oscillations, Vol. IV, Princeton University Press, New Jersey, 1958, pp. 1-18.

[15] Y. Kuang, B. G. Zhang, and T. Zhao, Qualitative analysis of a nonautonomous nonlinear delay differential equation, Tohoku Math. J. (2) 43 (1991), no. 4, 509-528.

[16] G. Ladas and C. Qian, Oscillation and global stability in a delay logistic equation, Dynam. Stability Systems 9 (1994), no. 2, 153-162.

[17] S. M. Lenhart and C. C. Travis, Global stability of a biological model with time delay, Proc. Amer. Math. Soc. 96 (1986), no. 1, 75-78.

[18] R. M. May, Time delay versus stability in population models with two or three trophic levels, Ecology 54 (1973), 315-325.

[19] S. C. Palaniswami and E. K. Ramasami, Nonoscillation of generalized nonautonomous logistic equation with multiple delays, Differential Equations Dynam. Systems 4 (1996), no. 3-4, 379-385.

[20] F. E. Smith, Population dynamics in daphnia magna and a new model for population growth, Ecology 44 (1963), 651-663. 


\section{Oscillation of a food-limited model}

[21] J. W.-H. So and J. S. Yu, On the uniform stability for a "food-limited" population model with time delay, Proc. Roy. Soc. Edinburgh Sect. A 125 (1995), no. 5, 991-1002.

[22] Z. Wang, J. S. Yu, and L. H. Huang, Nonoscillatory solutions of generalized delay logistic equations, Chinese J. Math. 21 (1993), no. 1, 81-90.

[23] E. M. Wright, A non-linear difference-differential equation, J. Reine Angew. Math. 194 (1955), 66-87.

[24] Z. Q. Yang, Necessary and sufficient conditions for oscillation of delay-logistic equations, J. Biomath. 7 (1992), no. 2, 99-109.

[25] B. G. Zhang and K. Gopalsamy, Oscillation and nonoscillation in a nonautonomous delay-logistic equation, Quart. Appl. Math. 46 (1988), no. 2, 267-273.

Leonid Berezansky: Department of Mathematics, Ben-Gurion University of The Negev, Beer-Sheva 84105, Israel

E-mail address: brznsky@cmath.bgu.ac.il

Elena Braverman: Department of Mathematics and Statistics, University of Calgary, 2500 University Drive N.W., Calgary, Alberta, Canada T2N 1N4

E-mail address: maelena@math.ucalgary.ca 


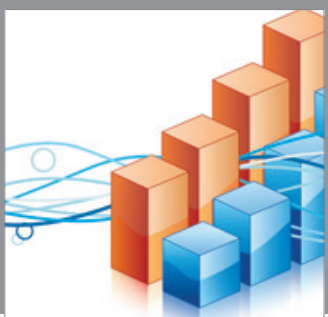

Advances in

Operations Research

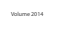

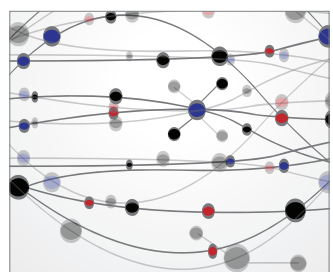

\section{The Scientific} World Journal
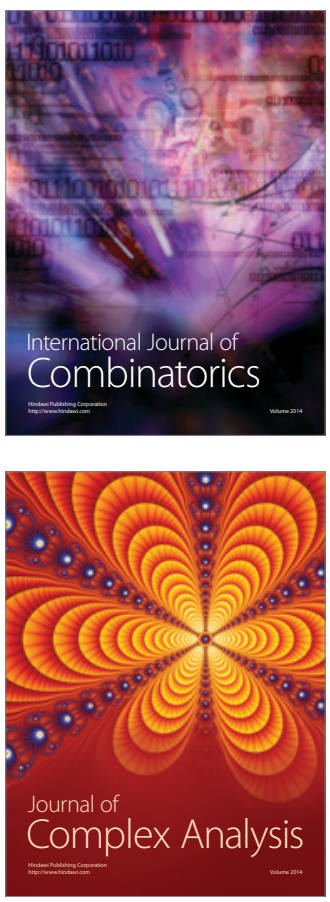

International Journal of

Mathematics and

Mathematical

Sciences
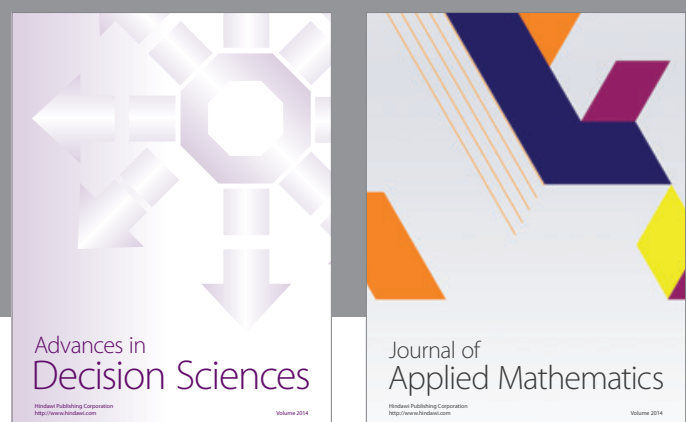

Journal of

Applied Mathematics
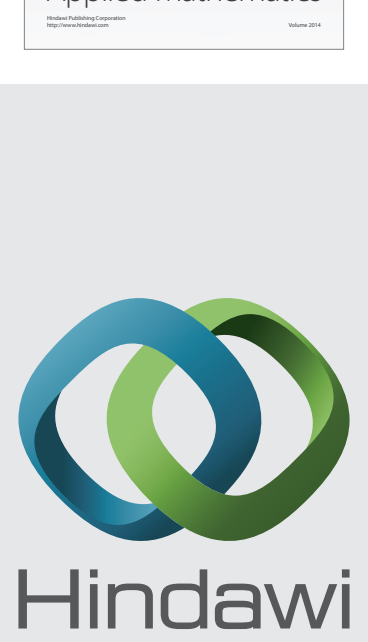

Submit your manuscripts at http://www.hindawi.com
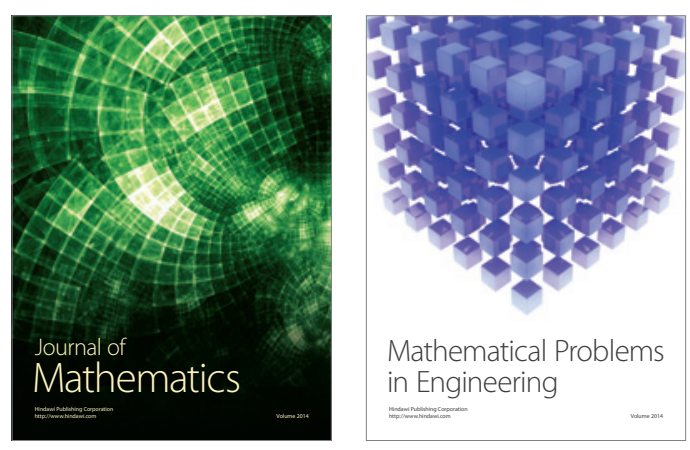

Mathematical Problems in Engineering
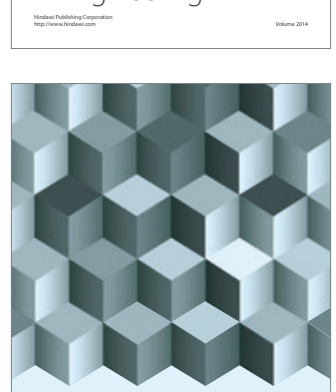

Journal of

Function Spaces
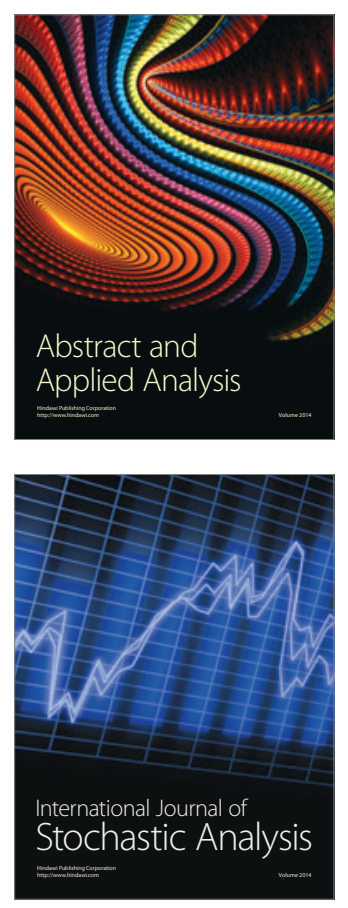

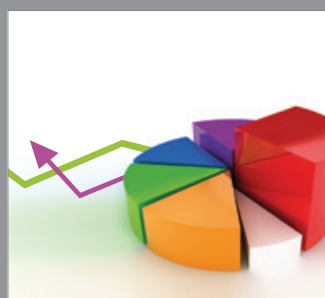

ournal of

Probability and Statistics

Promensencen
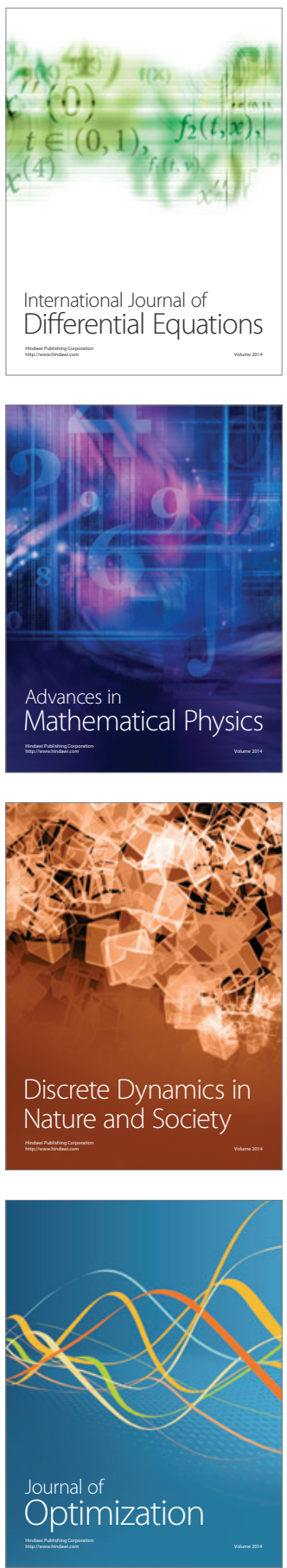